Apidologie, 1970, 1 (2), 143-155.

\title{
LA STABILISATION DU MIEL DE CALLUNE AU MOYEN D'UN TRAITEMENT THERMIQUE
}

\author{
Die Stabilisierung des Heide (Calluna)-Honigs durch \\ Wärmebehandlung
}

Pierre LAVIE et Michel GONNET

Station expérimentale d'Apiculture, Centre de Recherches d'Avignon, 84 - Montfavet

Institut national de la Recherche agronomique

\begin{abstract}
SUMMARY
STABILISATION OF HEATHER HONEY BY MEANS OF HEAT TREATMENT
\end{abstract}

Pasteurisation is a standard practice with honeys of normal viscosity. On the other hand this operation, said to be impossible, has never been tried on non-Newtonian honey (with abnormal viscosity). In spite of the thixotrophy of heather honey the authors have tried to stabilise it by means of a " managed " pasteurisation, the object being to kill the yeasts. Heat treatment has proved possible and the many observations made have allowed the physico-chemical and biological modifications undergone by the honey during treatment to be assessed.

\section{RESUMÉ}

La pasteurisation est d'une pratique courante en ce qui concerne les miels à viscosité normale. Par contre cette opération, réputée irréalisabłe, n'avait jamais été tentée sur un miel non newtonien (dont la viscosité est anormale). Malgré la thixotropie du miel de callune, les auteurs ont tenté de le stabiliser par une pasteurisation " ménagée " afin de détruire les levures. Le traitement thermique s'est révélé réalisable et de nombreux contrôles ont permis d'évaluer les modifications physicochimiques et biologiques subies par le mieł au cours des différentes phases de l'opération.

\section{INTRODUCTION}

Les miels de callune, à cause de leurs caractéristiques physico-chimiques, ont toujours posé des problèmes complexes aux apiculteurs et aux négociants européens, que ce soit pour l'extraction, le conditionnement ou le stockage. En effet 
ces miels récoltés par l'abeille sur la callune (Calluna vulgaris Salis), possèdent des propriétés physiques tout à fait différentes de celles des autres miels. La principale caractéristique de ces miels réside en une teneur en protéines voisine de 1 à $2 \%$, qui leur confère une certaine thixotropie. Ces miels possèdent une viscosité qui est variable dans le temps, et ceci sans modification ni de la température, ni de la teneur en eau. Ils sont susceptibles de passer de l'état de gel à l'état de sol sous l'action d'une agitation mécanique et de reprendre ensuite peu à peu l'état de gel. Notons cependant, sans entrer dans le détail, que le miel de callune n'est pas vraiment thixotrope dans le sens que les physiciens donnent à ce mot puisque, même après agitation, il n'y a jamais retour à l'état de sol parfait mais seulement disparition partielle de l'état de gel. Ceci se complique encore du fait qu'un miel de callune est rarement pur et qu'il est mélangé au cours de l'extraction en proportions variables avec des miels à viscosité normale. Plus le miel est pur callune et plus sa viscosité est anormale. On sait également par expérience que le vieillissement des miels de callune et leur chauffage accentuent les phénomènes de gélification. Ces caractéristiques ont fait l'objet de travaux détaillés : PriceJONES (1953).

Les anomalies du miel de callune interdisent de le traiter comme les miels à viscosité normale ou miels " newtoniens ". En effet, la viscosité d'un miel newtonien est liée à sa teneur en eau et à sa température; un chauffage compris entre $35^{\circ}$ et $40^{\circ}$ favorise donc les opérations d'extraction par la force centrifuge, de filtration, de décantation, de pompage et de circulation dans des canalisations. Par contre il est toujours difficile de manipuler le miel de callune même à 35 ou $40^{\circ}$ et une élévation de température plus importante ne résout pas les problèmes car elle entraîne une coagulation des protéines qui aboutit après repos à la constitution d'un gel irréversible.

Dans la pratique il est donc difficile de filtrer et de décanter les miels de callune. Lors de la décantation, les bulles d'air incluses lors de l'extraction et de la filtration ne remontent pas à la surface. Elles restent prisonnières dans la masse en grand nombre, ce qui oxygène parfaitement le miel et favorise le développement des levures, donc les fermentations. Il s'ajoute à cet inconvénient que tous les miels de callune sont riches en eau, puisque leur pourcentage normal d'humidité est de 20 à $25 \%$. Le danger de fermentation est donc accru et, de fait, les miels de callune fermentent assez facilement après l'extraction. Ces miels sont donc difficiles à stocker et ils perdent rapidement leur valeur commerciale au cours de la conservation. On peut empêcher la fermentation par un abaissement de température mais la conservation au froid est coûteuse et ne résoud pas complètement la question. En effet, s'il est possible de stocker le miel à basse température, il faut ensuite le conditionner et le commercialiser; c'est à ce moment que se présentent les inconvénients dus à la fermentation du produit. La déshydratation partielle du miel de callune, qui poserait des problèmes à l'échelon industriel, ne donnerait pas non plus les résultats escomptés. Louveaux a montré en 1966 que des miels de callune ramenés artificiellement à $18 \%$ d'eau perdaient leur saveur particulière et devenaient franchement mauvais. L'élimination des bulles d'air de la masse du miel ne serait pas techniquement impossible mais demanderait la mise en œuvre de moyens assez coûteux et n'empêcherait pas de toute manière les levures de se développer dans un miel aussi riche en humiditié. 
Il nous a alors semblé qu'il serait intéressant de détruire les levures par un traitement thermique approprié et si possible en utilisant les échangeurs thermiques d'une unité de pasteurisation conçue pour le traitement des miels normaux. En effet si l'opération était réalisable, le même matériel de base pourrait, dans une coopérative ou chez un négociant, être utilisé à deux fins : stabilisation physique des miels de viscosité normale et stabilisation biologique et physique des miels à tendance thixotropique. A priori, l'opération était réputée irréalisable du fait de la thixotropie du miel de callune qui devait interdire la circulation dans les échangeurs thermiques principalement lors du refroidissement. Cependant, étant donné 1'importance du problème, notamment dans le sud-ouest de la France nous avons essayé malgré tout de stabiliser le miel de callune en utilisant l'unité de pasteurisation de la station expérimentale d'apiculture de Montfavet. Les miels nécessaires aux essais nous ont été fournis au cours des années 1966 et 1967 par la Coopérative des propucteurs de miels du sud-ouest à Carcarès Sainte-Croix (Landes) qui était intéressée au premier chef par cette étude. Les buts poursuivis furent les suivants :

$1^{0}$ destruction des levures par élévation de température;

$2^{\circ}$ obtention d'un miel dont les caractéristiques gustatives, physico-chimiques et biologiques soient compatibles avec une bonne commercialisation.

\section{MATERIEL ET METHODES}

\section{A. - Le pasteurisateur}

Nous nous sommes servis du pasteurisateur A.P.V. de laiterie adapté à la pasteurisation du miel, appareil que nous avons décrit longuement dans deux mémoires précédents (LAvrE, 1961 et Gonnet, Lavie, Louveaux, 1964). L'installation est classique et ne comporte des dispositifs spéciaux que dans le circuit de refroidissement. La première expérience fut tentée avec ce matériel destiné à traiter des miels courants, sans aucune adaptation spéciale. $1200 \mathrm{~kg}$ de miel de callune très pur, après refonte à $40^{\circ} \mathrm{C}$ pendant deux jours et filtrage, furent maintenus à $40^{\circ} \mathrm{C}$ en préchauffage et enfin portés à $70^{\circ} \mathrm{C}$ dans l'échangeur thermique. Comme prévu ce miel fluidifié et mis à l'état de "Sol " par les différentes manipulations et par le pompage n'a cependant pas pu franchir le circuit de refroidissement à $40^{\circ} \mathrm{C}$. Nous avons alors modifié l'installation et laissé sortir le miel après son chauffage à $70^{\circ} \mathrm{C}$ sans le refroidir immédiatement. Malgré l'absence d'un refroidissement rapide les contrôles nous ont indiqué que l'invertase ne se trouvait pas détruite totalement, que les levures étaient tuées et que l'aspect final du miel traité était très satisfaisant. Cet essai " pour voir " nous avait donc donné quelques indications positives et nous avons décidé de reprendre cette étude avec un matériel modifié afin d'obtenir un traitement respectant les constituants du miel. Compte tenu de l'essai précédent nous avons chauffé le miel à $70^{\circ} \mathrm{C}$ par la méthode déjà décrite, mais nous l'avons refroidi par passage dans des tubes en pyrex à large section afin d'éviter le colmatage. Ces tubes de $40 \mathrm{~mm}$ de diamètre, montés en forme de serpentin, étaient immergés dans une cuve à circulation d'eau froide (fig. 1 et fig. 2). Le dispositif nous permettait de faire varier la température de chambrage et le temps de refroidissement. En général, le miel a été porté à $70^{\circ} \mathrm{C}$ dans l'échangeur thermique à plaques, chambré 5 à 6 minutes à cette température et refroidi dans le serpentin de grosse section jusqu'à $57^{\circ} \mathrm{C}$ ou $52^{\circ} \mathrm{C}$. La vitesse de passage du miel dans l'appareil se situait aux environs de $160 \mathrm{à} 180 \mathrm{~kg} /$ heure. Le circuit de refroidissement a représenté $4,5 \mathrm{~m}$ ou $8,5 \mathrm{~m}$ de tubes immergés dans un bac de 500 litres où de l'eau à $17-18^{\circ}$ circulait à la vitesse de $2 \mathrm{~m}^{3} /$ heure. A la sortie du pasteurisateur, à $52^{\circ} \mathrm{C}$ ou $57^{\circ} \mathrm{C}$, le miel était traité de deux manières. Dans le premier cas, il était conditionné immédiatement en pots de $500 \mathrm{~g}$ parfaitement propres que l'on plaçait rapidement en chambre froide à $4{ }^{\circ} \mathrm{C}$, de manière à obtenir un refroidissement rapide. Dans le deuxième cas il était versé en vrac dans des fûts de $300 \mathrm{~kg}$ afin d'obtenir un refroidissement lent et d'étudier l'influence d'un choc thermique plus important. Cependant, il faut noter qu'il ne s'agissait pas d'une vraie pasteurisation et que le miel de callune subissait seulement un traitement thermique destiné à détruire les levures et par là même à le stabiliser d'où le titre du présent mémoire. 


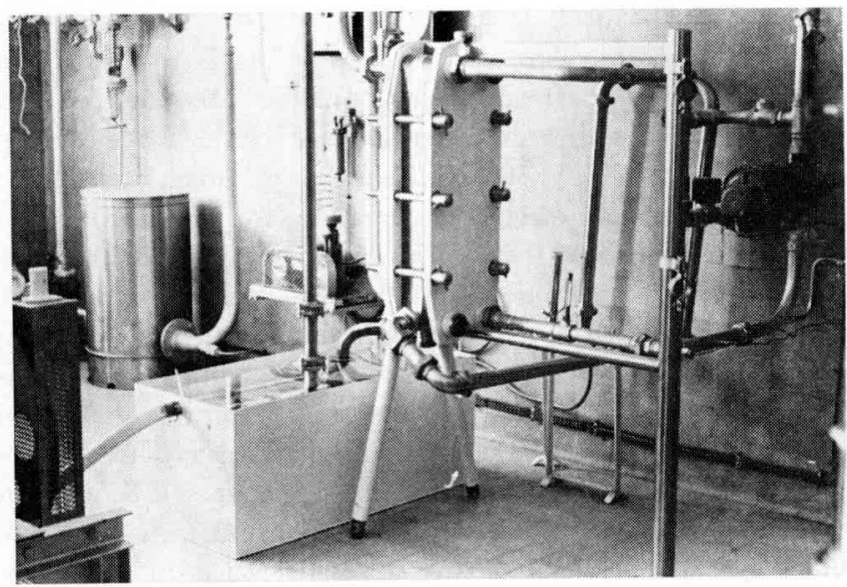

Fic. 1. - Vue d'ensemble du pasteurisateur

Au centre l'échangeur thermique. En bas et à gauche le bac de refroidissement.

Aвв. 1. - Gesamtansichı des Pasteurisier-Apparates

In der Mitte der Wärme-Austauscher. Cnten und links der Abkühlungstrog.

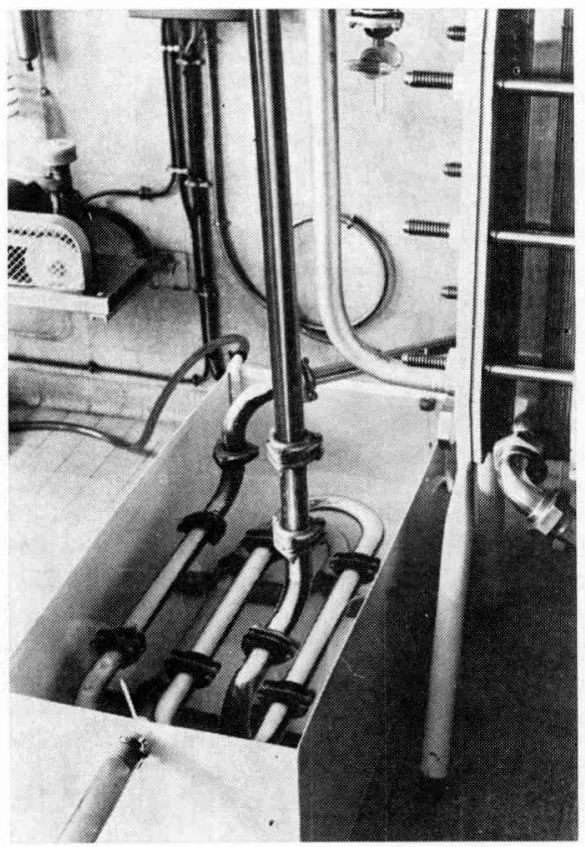

Fic. 2. - Détail du bac de refroidissement miel.

On aperçoit le serpentin formé de tubes de pyrex de $40 \mathrm{~mm}$ de diamètre dans lequel circule le

Aвв. 2. - Teil des Abkühlungstroges

Man sieht die Schlange aus Pyrex-Rohren mit $40 \mathrm{~mm}$ Durchmesser, in denen der Honig zirkuliert. 


\section{B. - Les miels traités}

Tous les miels, livrés en fûts de $300 \mathrm{~kg}$ représentant au total environ $15000 \mathrm{~kg}$, provenaient des Landes et se répartissaient en 5 lots différents. Le premier lot consistait en un miel de callune très pur dont la teneur en eau était de $21 \%$. Le lot $\mathbf{n}^{\circ} 2$ correspondait à un mélange de miel de callune avec un autre miel à viscosité normale en proportion mal définie et d'une teneur en eau de $20,5 \%$ Le lot $n^{\circ} 3$ était un miel de callune riche en eau $(24,6 \%)$ et ayant subi un début de fermentation. Les lots $n^{\circ} 4$ et $n^{\circ} 5$, de teneur en eau voisine $(21,6$ et $22,7 \%$ ) étaient des miels de callune presque purs. Sur l'ensemble de ces miels un certain nombre d'opérations ont été effectuées : chauffage et refonte des fûts de miels, préchauffage avant la " pasteurisation ", chauffage à $70^{\circ} \mathrm{C}$ pendant 5 à 6 minutes, refroidissement à $52^{\circ} \mathrm{C}$ ou à $57^{\circ} \mathrm{C}$ à la sortie, mise en pots ou en fûts. A chaque opération un prélèvement d'échantillons était effectué afin de procéder à un certain nombre de contrôles. Le tableau 1 donne le détail de 19 opérations qui ont donné lieu à des essais précis.

\section{C. - Les analyses et les contrôles effectués}

Les mesures d'humidité étaient effectuées au refractomètre Abbe (marque Zeiss). La teneur en eau était obtenue en utilisant les tables de Chataway (1954) et en procédant aux corrections de températures.

L'hydroxymethylfurfural (H.M.F.) était dosé par la méthode quantitative de WINKLER (1955). Les résultats sont exprimés en milligramme par $100 \mathrm{~g}$ de miel.

L'invertase était dosée suivant la méthode de Hadonn et Zunchen (1962). Les résultats sont exprimés en milligramme de saccharose hydrolysé en une heure par l'invertase contenue dans $100 \mathrm{~g}$ de miel.

L'amylase a été dosée suivant la méthode de Shade, Marsch et Eckert (1958). Les résultats sont exprimés en millilitre d'amidon (solution à $1 \%$ ) hydrolysé en une heure par l'amylase contenue dans $1 \mathrm{~g}$ de miel.

Le dosage de l'inhibine, mis au point par Dold et WITZENHausen (1955) et repris par GonNET et Lavie (1960) a été appliqué sans modification. La souche bactérienne de référence est Bacillus subtilis Caron. Les lectures sont effectuées 24 heures après l'ensemencement et les résultats sont exprimés par une note de 0 à 5 avec une approximation de 0,25 .

Fermentation. Nous avons mis au point et utilisé pour quelques échantillons un test simple qui permet de reconnaître si un miel contient ou non des microorganismes vivants susceptibles de le faire fermenter. On introduit de petites quantités de miel dans des flacons stériles et on les dilue avec de l'eau stérile jusqu'à des teneurs en eau allant de la teneur initiale à $60 \%$. Les flacons bouchés avec un tampon d'ouate stérile sont porté 3 à l'étuve à $25^{\circ} \mathrm{C}$. On observe le développement des levures qui se traduit par un trouble du milieu et un dégagement gazeux au bout de $70,90,160,240$ et 300 heures.

\section{RÉSULTATS}

La présentation des principaux résultats obtenus a nécessité l'établissement de trois tableaux qui feront l'objet d'un bref commentaire. Tous les dosages et toutes les notations ont été faits selon les principes exposés au chapitre précédent. Il conviendra donc de se reporter à ce chapitre pour la compréhension des résultats de même que l'on consultera le tableau 1 qui regroupe toutes les données relatives aux échantillons des miels étudiés.

Tout d'abord ce qu'il faut préciser c'est que l'opération de stabilisation thermique avec refroidissement dans des conditions bien définies est possible. Le miel de callune circule dans le circuit sans difficulté; l'enregistrement de la température pendant le chauffage à $70^{\circ} \mathrm{C}$ montre la régularité du passage du miel.

Le but principal recherché a été atteint. En effet, l'ensemble de l'opération, chauffage à $70^{\circ} \mathrm{C}$ pendant 5 minutes suivie d'un refroidissement à $52^{\circ} \mathrm{C}$, détruit 


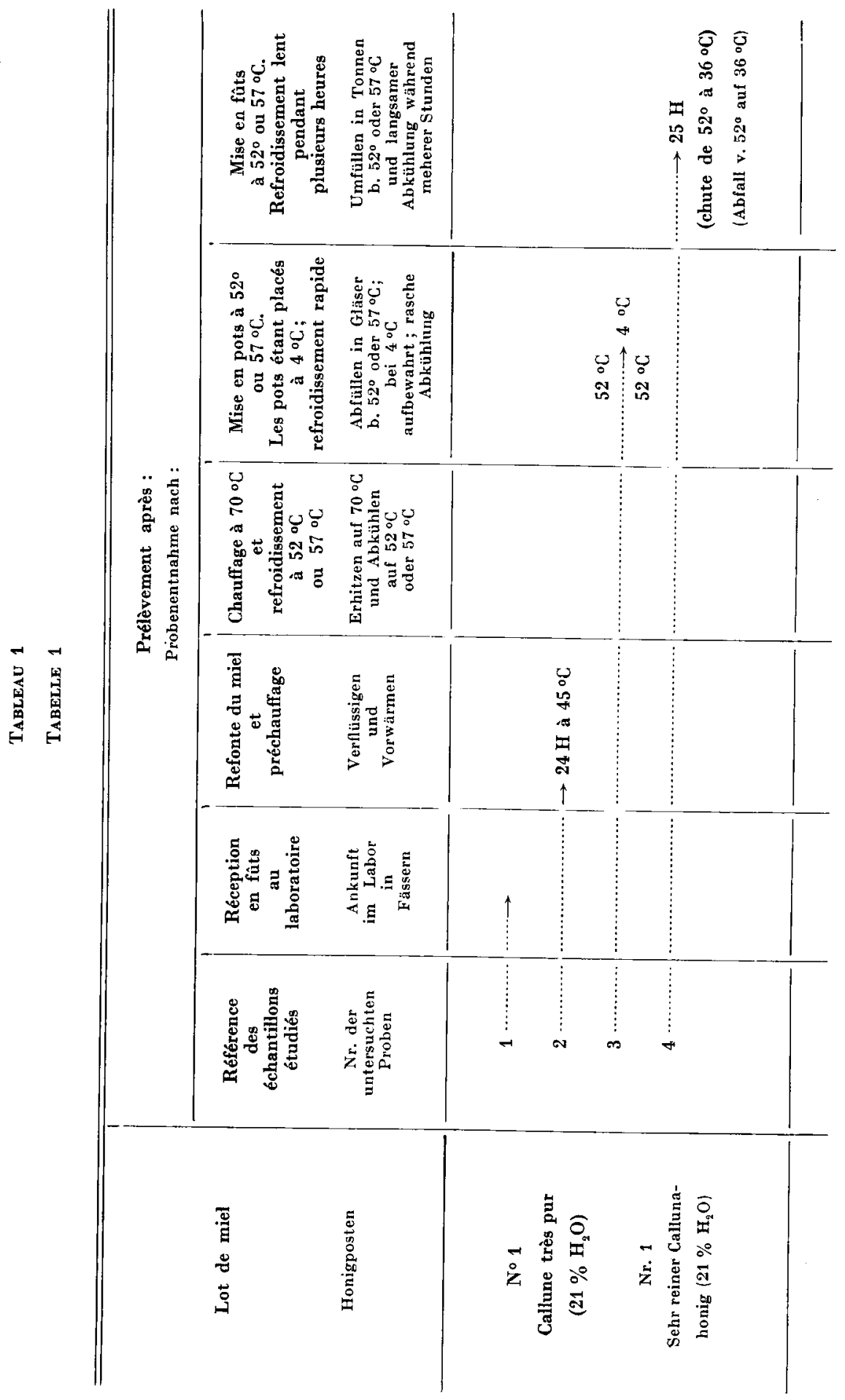


STABILISATION DU MIEL DE CALLUNE
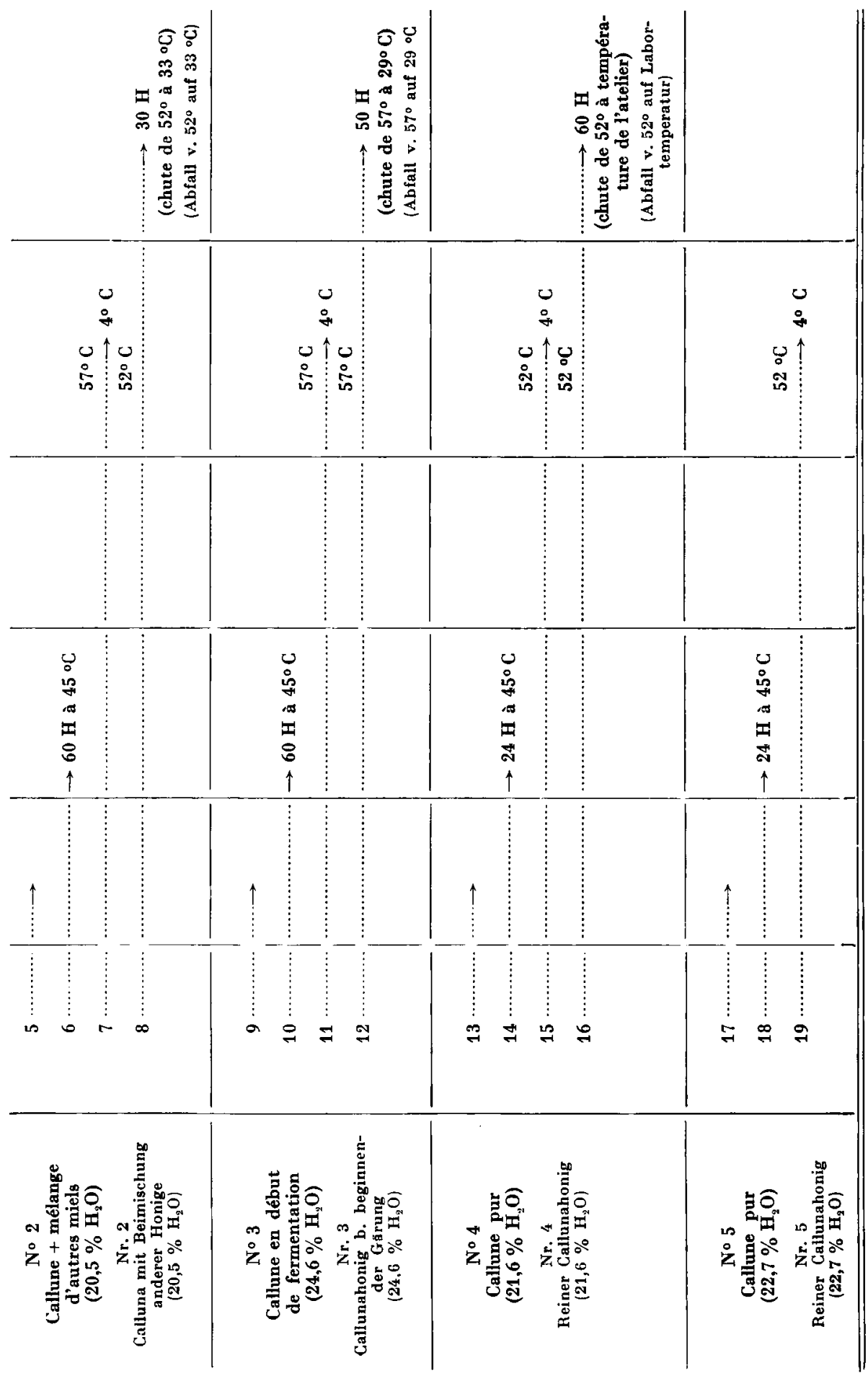
les levures présentes dans le miel tandis que les miels non traités ou seulement préchauffés fermentent normalement. Même après 12 jours à $25^{\circ} \mathrm{C}$ et à des dilutions atteignant $60 \%$ d'eau les miels chauffés à $70{ }^{\circ} \mathrm{C}$ ne fermentent pas. Le tableau 2 donne le détail de ces résultats.

TABL. 2. - Epreuve de fermentation des échantillons de miel avant et après stabilisation

TAB. 2. - Untersuchung der Honigproben auf Gürung vor und nach dem Stabilisieren

\begin{tabular}{|c|c|c|c|c|c|c|}
\hline \multirow{2}{*}{$\begin{array}{c}\text { Référence des échan- } \\
\text { tillons } \\
\text { Nr. der Proben }\end{array}$} & \multirow{2}{*}{$\begin{array}{l}\text { Dilution du miel à } \\
\text { (1) } \\
\text { Verdünnen des } \\
\text { Honigs auf (1) }\end{array}$} & \multicolumn{5}{|c|}{$\begin{array}{c}\text { Fermentation après } \\
\text { Gären nach }\end{array}$} \\
\hline & & $70 \mathrm{H}$ & $90 \mathrm{HI}$ & $160 \mathrm{H}$ & $240 \mathrm{H}$ & $300 \mathrm{H}$ \\
\hline \multirow{5}{*}{$\begin{array}{c}1,5,9,13,17 \\
\text { Miels non traités } \\
\text { Nicht behandelte Honige }\end{array}$} & $\begin{array}{c}\stackrel{0}{\%} \\
21 \stackrel{\text { à }}{2}\end{array}$ & $\cdots$ & - & -- & $\cdots$ & - \\
\hline & 30 & $\cdots$ & - & -- & + & \\
\hline & 40 & - & \pm & + & & \\
\hline & 50 & t & & & & \\
\hline & 60 & + & & & & \\
\hline \multirow{5}{*}{$\begin{array}{l}2,6,10,14,18 \\
\text { Miels pré-chauffés } \\
\text { Vorgewärmte Honige }\end{array}$} & 21 à 24 & & -. & - & $\cdots$ & - \\
\hline & 30 & & $\ldots$ & - & - & \pm \\
\hline & 40 & - & - & + & & \\
\hline & 50 & -. & $t$ & & & \\
\hline & 60 & $\stackrel{+}{+}$ & & & & \\
\hline \multirow{5}{*}{$\begin{array}{l}3,7,11,15,19 \\
\text { Miels stabilisés } \\
\text { Stabilizierte Honige }\end{array}$} & 21 à $24 \%$ & - & & $\cdots$ & $\cdots$ & - \\
\hline & 30 & -- & -- & - & -. & 一 \\
\hline & 40 & -- & -- & $\cdots$ & - & - \\
\hline & 50 & - & - & - &.- & - \\
\hline & 60 & - & - - & - & - - & - \\
\hline
\end{tabular}

+ : échantillon fermenté $=$ gegorene Probe.

\pm : échantilion en début de fermentation $=$ beginnende Gärung $d$. Probe.

- : échantilion non fermenté $=$ nicht gegorene Probe.

(1) Le premier pourcentage est celui de la concentration initiale en eau. Die erste Prozentzahl bezicht sich auf den anfänglichen Wassergehalt. 
L'ensemble du tableau 3 montre les résultats d'analyses concernant certains constituants du miel avant et après les différentes opérations subies par le miel de callune.

On remarque tout d'abord qu'il n'y a pas de production d'HMF en quantité appréciable après le traitement. Toutefois un dosage fin est difficile à cause du trouble irrégulier dû aux protéines du miel.

En ce qui concerne l'inhibine, sa destruction est faible et elle n'est pas toujours constante.

L'amylase est détruite en partie mais faiblement et beaucoup plus dans les lots où la teneur en eau est la plus élevée. La chute de la teneur en amylase a principalement lieu dès le préchauffage ; ensuite le chauffage à $70^{\circ}$ ne modifie pas les chiffres. Cependant on remarquera qu'un miel du lot no 3 (prélèvement $n^{\circ} 12$ ) maintenu à une température élevée pendant un temps assez long a perdu beaucoup plus d'amylase que les autres.

Par contre nous savions que l'invertase serait plus vulnérable. En effet l'ensemble des manipulations de refonte du miel en fûts de $300 \mathrm{~kg}$, de préchauffage et de traitement dans les conditions indiquées provoquent une perte de 45 à $50 \%$ de l'invertase initiale. La destruction a lieu par paliers comme l'illustrent les tableaux 3 et 4 . Une première destruction a lieu après le préchauffage; elle est généralement

TABL. 3. - Résultats d'analyses concernant les miels de callune avant et après stabilisation

TAв. 3. - Analysenergebnisse von Heidehonigen vor und nach dem Stabilisieren

\begin{tabular}{|c|c|c|c|c|c|}
\hline $\begin{array}{c}\text { Référence } \\
\text { de l'échantillon } \\
\text { Nr. der Proben }\end{array}$ & $\mathrm{H}_{2} \mathrm{O}$ & H.M.F & Invertase & Amylase & Tnhibine \\
\hline 1 & $21 \%$ & 0,60 & 16,50 & 50 & 4 \\
\hline 2 & - & 0,60 & 13,30 & 47 & 3,75 \\
\hline 3 & - & 0,90 & 10,70 & 47 & 3 \\
\hline 4 & - & 0,90 & 9,0 & 47 & 3 \\
\hline 5 & $20,5 \%$ & 0,60 & 16,70 & 49 & 4 \\
\hline 6 & - & 0,80 & 8,70 & 45 & 4 \\
\hline 7 & - & 0,80 & 7,00 & 43 & 3,25 \\
\hline 8 & - & 0,90 & 7,00 & 44 & 3 \\
\hline 9 & $24,6 \%$ & 0,20 & 16,70 & 47 & 4 \\
\hline 10 & - & 0,60 & 8,70 & 43 & 4 \\
\hline 11 & - & 0,90 & 6,10 & 43 & 3,50 \\
\hline 12 & - & 0,90 & 2,00 & 35 & 3,50 \\
\hline 13 & $21,6 \%$ & 0,40 & 16,70 & 52 & 4 \\
\hline 14 & - & 0,40 & 13,40 & 47 & 4 \\
\hline 15 & - & 0,80 & 10,70 & 47 & 4 \\
\hline 16 & - & 0,80 & 8,70 & 47 & 4 \\
\hline 17 & $22,7 \%$ & 0,40 & 16,70 & 48 & 4. \\
\hline 18 & - & 0,60 & 12,40 & 44 & 4 \\
\hline 19 & - & 0,90 & 8,70 & 44 & 4 \\
\hline
\end{tabular}


P. LAVIE, M. GONNET

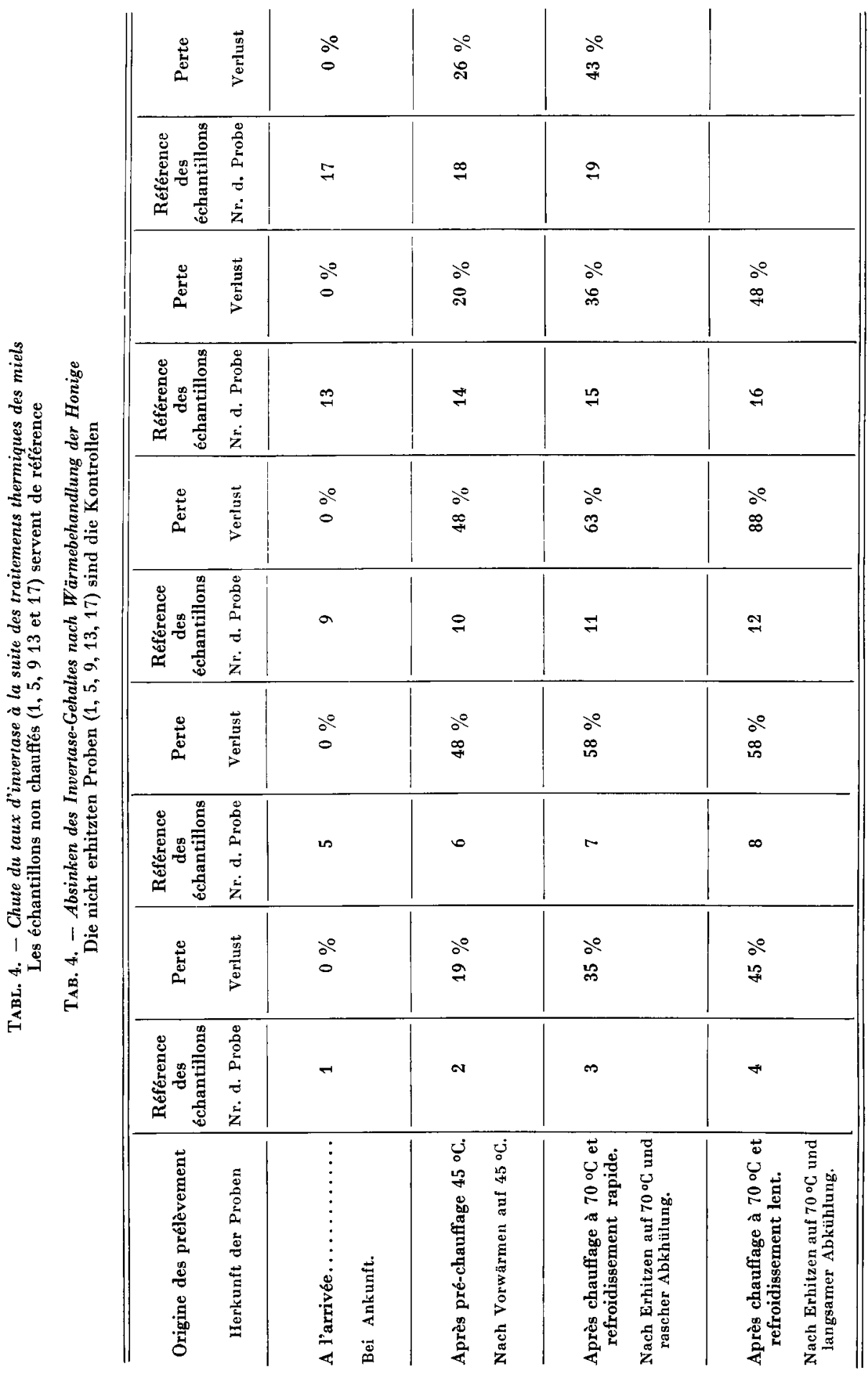


importante de 19 à $48 \%$, mais elle est très variable suivant la durée et l'intensité du préchauffage. On constate que pour les lots 2 et 3 l'opération fut trop longue. La seconde chute du taux de l'invertase a lieu pendant le chauffage à $70^{\circ} \mathrm{C}$ (de 10 à $22 \%$ ) ; elle est moins importante que la perte due au préchauffage mais elle est également variable. L'échantillon de miel $\mathrm{n}^{0} 18$, pasteurisé pendant 6 minutes accuse la destruction la plus forte $(22 \%)$. Enfin le temps de refroidissement des miels traités influe beaucoup. Entre un refroidissement rapide (miels mis en pots et placés à $4{ }^{\circ} \mathrm{C}$ )et un refroidissement lent - (miel mis en fûts et laissés à refroidir à la température ordinaire) la destruction de l'invertase varie de 0 à $25 \%$. Ainsi les échantillons 7 et 8 possèdent la même teneur finale en invertase bien qu'ils aient été traités différemment. Le $\mathrm{n}^{\circ} 7$ a été refroidi à $57^{\circ} \mathrm{C}$ et placé en pots à $4{ }^{\circ} \mathrm{C}$, tandis que le $\mathrm{n}^{\circ} 8$ a été mieux refroidi au cours de la pasteurisation $\left(52^{\circ} \mathrm{C}\right)$ mais placé ensuite en fûts de $300 \mathrm{~kg}$ à température ordinaire pour y subir un refroidissement très lent ( 30 heures pour passer de $52^{\circ} \mathrm{C}$ à $\left.33^{\circ} \mathrm{C}\right)$. Il est donc préférable de refroidir les miels à $52^{\circ} \mathrm{C}$ au lieu de $57^{\circ} \mathrm{C}$ après le chauffage à $70^{\circ} \mathrm{C}$. L'opération est suffisante pour la stabilité'du miel et les diastases sont beaucoup moins dégradées. On remarquera encore que la chute de la teneur en diastases la plus importante s'est produite dans le lot $n^{\circ} 3$. Ceci peut s'expliquer par une acidité plus forte que la normale et que nous avons mesurée. Cet excès d'acidité est certainement dû au début de fermentation du miel.

Enfin nous avons eu la surprise d'obtenir un miel stabilisé dont l'aspect est très satisfaisant et valorise le produit. Le miel de callune traité prend après refroidissement en pots la texture d'un gel parfait emprisonnant de multiples bulles d'air. Le produit final est d'une présentation irréprochable et il s'est vendu facilement au détail sur le marché français, chose impossible par les procédés ordinaires.

\section{CONCLUSION}

Cette étude a démontré qu'il était possible de traiter les miels de callune au moyen d'un échangeur thermique et de le stabiliser du point de vue biologique en détruisant les levures. Cette stabilité excède six mois, ce qui correspond aux conditions normales de distribution et de vente, sous réserve de conditionner dans des récipients propres afin d'éviter un réensemencement. Nous avons remarqué également que si le miel traité est mis directement en fût à $52^{\circ} \mathrm{C}$ il est possible de le refondre pour le conditionner plus tard mais cette pratique n'est guère recommandable. En effet, les refontes et réchauffements successifs produisent des effets néfastes qui s'additionnent à chaque manipulation. D'autre part, le miel mis en fût de $300 \mathrm{~kg}$ à la sortie du pasteurisateur met encore longtemps avant d'atteindre la température ambiante alors que s'il est mis directement en pots il refroidit assez vite. Il faut remarquer également que les miels de callune sont naturellement riches en diastases et que la perte subie en amylase et en invertase lors du passage à $70^{\circ} \mathrm{C}$ pendant 5 minutes est finalement très acceptable. Les miels traités avec précaution et refroidis à $52^{\circ} \mathrm{C}$ à la sortie de l'appareil possèdent donc une teneur en diastases suffisante pour être commercialisés sans difficultés sur le marché européen car ils restent largement au-dessus des normes internationales généralement admises. En fait seule l'invertase subit une perte très sensible pendant toute l'opé- 
ration. Cependant, certaines précautions permettent de réduire considérablement cette perte et notre expérience nous permet d'insister plus particulièrement sur deux points; il faut :

$1^{\circ}$ essayer de réaliser un préchauffage (et refonte s'il y a lieu) aussi rapide et modéré que possible;

$2^{\circ}$ essayer d'obtenir après la pasteurisation un refroidissement aussi rapide que possible.

En effet comme le montrent les résultats précédents le passage du miel à $70{ }^{\circ} \mathrm{C}$ pendant 5 minutes n'est pas la cause essentielle de la chute du taux de l'invertase. Quant aux autres constituants du miel ils ne sont pas touchés sensiblement par l'ensemble du traitement thermique.

La méthode reste perfectible. Il est sans doute possible par exemple de remplacer le serpentin de refroidissement par un échangeur thermique dont les plaques seraient plus écartées. Il serait préférable d'autre part de travailler sur des lots bien mélangés avant le traitement pour assurer une bonne régularité de la circulation du miel dans les canalisations.

\author{
Reçu pour publication en janvier 1970. \\ Eingegangen im Januar 1970.
}

\title{
ZUSAMMENFASSUNG
}

Heidehonige (Calluna vulgaris Salisb.) haben ihrer physikochemischen Eigenschaften wegen Imker und Honighändler immer wieder vor besondere Probleme gestellt; sei es bei der Schleuderung, dem Abfütlen oder bei der Aufbewahrung. Diese Honige enthalten einen hohen Prozentsatz an Proteinen und zeichnen sich durch eine aussergewöhnliche Thixotropie aus. Ausserdem ist ihr Wassergehalt viel höher als bei anderen Honigen. Nach der Schleuderung weist der Honig eine Unzahl an Luftblasen auf, die die Gärung während der Aufbewahrungszeit begünstigen.

In unserer Arbeit haben wir versucht, mit angemessener Wärmebehandlung die im Honig vorhandenen Hefen zu zerstören. Pasteurisieren, wie es bei anderen Honigen angewandt wird, erschien uns bei Calluna-Honigen wegen ihrer Thixotropie nicht angebracht. Mit Hilfe eines PlattenWärmeaustauschers mit abgeändertem Abkühlungssystem haben wir eine schonende Pasteurisierung erreicht. Bei deiser Behandlung, einem nicht eigentlichen Pasteurisieren, wird der Honig 5 Min auf $70^{\circ} \mathrm{C}$ erhitzt und beim Verlassen des Apparates auf $52^{\circ} \mathrm{C}$ abgekühlt. $15000 \mathrm{~kg}$ Heidehonig unterschiedlichen Wassergehaltes und von verschiedenem Reinheitsgrad wurden auf diese Weise behandelt. Vor jedem Arbeitsgang wurden Proben entnommen und verschiedenen Prüfungen unterzogen. Der Wassergehalt wurde mit dem Abbe-Refraktometer, der HMF-Gehalt quantitativ nach WINKLER bestimmt. Die Invertase-Bestimmung erfolgte nach der Methode von HADORN und ZuRCHER Zur Bestimmung des Amylsagehaltes wurde die Methode von Shade, Marsch und Eckert benutzt. Die Inhibine bestimmten wir mit der von GonNET und LAvIE abgewandelten Methode nach DoLD und Wiztenhausen. Ausserdem entwickelten wir einen einfachen Test zur Feststellung lebender, eine Gärung hervorrufender Mikroorganismen im Honig.

Die Ergebnisse sind aus den Tabellen ersichtlich. 
Zusammenfassend Kann gesagt werden :

Unter den beschriebenen Bedingungen ist die Stabilisierung des Heidehonigs durch Erhitzen und Abkühlen möglich. Das gesteckte Ziel wurde also erreicht. Die Hefen werden zerstört, während nicht behandelte oder nur vorgewärmte Honige gären. Der Hydroxymethylfurfurol - Gehalt wird durch die Behandlung nicht wesentlich erhöht. Inhibine und Amylasen nur unwesentlich beeinträchtigt. Dagegen verringert sich die in diesen Honigen vorhandene Invertase um 45-50\%. Man muss allerdings bedenken, dass das Vorwärmen vor dem eigentlichen Pasteurisieren sich schon sehr nachteilig auf die Invertase auswirkt. Schliesslich ist das Aussehen der behandelten Honige sehr zufriedenstellend. Nach der Abkühlung hat der Honig die Beschaffenheit eines unzählige Luftbläschen einschliessenden, sehr festen Gelees. Zum Schluss möchten wir noch darauf hinweisen, dass bei der Wärmebehandlung des Heide-(Calluna)-Honigs folgende Bedingungen erfüllt sein sollten :

$1^{\circ}$ Das Vorwärmen (und gegebenenfalls eine Verflüssigung) muss so rasch und so schonend wie möglich vor sich gehen, da bei langanhaltender Erwärmung die Schädigenden Wirkungen sich verstärken.

$2^{\circ}$ Die Abkühlung nach dem Pasteurisieren muss so rasch und so wirksam wie möglich erfolgen, wobei es ratsam erscheint, den Honig in kleine Gefässe abzufüllen.

\section{RÉFÉRENCES BIBLIOGRAPHIQUES}

Chataway H. D., 1954. In ABC and XYZ of bee culture, 438, Root Co, Medina (Ohio), U.S.A.

Dold H., Witzenhausen R., 1955. Ein Verfahren zur Beurteilung der örtlichen inhibitorischen (Keimvermehrungshemmenden) Wirkung von Honigsorten verschiedener Herkunft. Z. Hyg., 141, 333-337.

Gonnet M., Lavie P., 1960. Influence du chauffage sur le facteur antibiotique des miels. Ann. Abeille, 3 (4), 349-364.

Gonnet M., Lavie P., Louveaux J., 1964. La pasteurisation des miels. Ann. Abeille, 7 (2), 81.102.

Hadorn H., Zurcher K., 1962. Zur Bestimmung der Saccharase-Aktivität in honig. Mitt Lebens. Hyg., 53 (I), 5-28.

LAviE P., 1961. L'appareillage pour la pasteurisation des miels à la station expérimentale d'apiculture de l'INRA. Symposium scientifique sur les questions relatives au miel 22 septembre 1961, Madrid.

Louveaux J., 1966. Essai de caractérisation des miels de Callune (Calluna vulgaris Salisb.). Ann. Abeille, 9 (4), 351-358.

Pryce-Jones J., 1953. The rheology of honey. In * Foodstuffs, their plasticity, fluidity and consis. tency ". Amsterdam.

Shade J., Marsch G., Eckert J., 1958. Diastase activity and hydroxymethylfurfural in honey and their usefulness in detecting heat alteration. Food Research, 23, 446-463.

WINKLER O., 1955. Beitrag zum Nachweis und zur Bestimmung von oxymethylfurfurol in Honlyr und Kunsthonig. Z. Unters. Lebensmittel, 102, 161-167. 